\title{
Experimental Study on Stabilization Subgrade Soil Soft with Fine Concrete Residue
}

\section{Estudio Experimental Sobre la Estabilización de Suelo de Subrasante Blando con Residuo de Concreto Fino}

I International Congress of Science and Technology Morona Santiago-CICTMS 2020

Corresponding Author:

Sara del Rocio Ochoa Averos rocioochoa_1996@hotmail.com

Published: 29 August 2021

Production and Hosting by Knowledge $E$

(c) Sara del Rocio Ochoa Averos and Julio César Bizarreta-Ortega. This article is distributed under the terms of the Creative Commons Attribution License, which permits unrestricted use and redistribution provided that the original author and source are credited.

\section{Sara del Rocio Ochoa Averos and Julio César Bizarreta-Ortega}

Universidad Federal de la Integración Latinoamericana, Instituto Latino-Americano de Tecnologia, Infraestrutura y Território, Foz de Iguazu, Brasil

\section{Abstract}

The reuse of construction and demolition waste (CDW) is a sustainable practice that in recent years has been investigated with great intensity around the world. Concrete waste are a part of the CDW, whose application in pavements is still under development. In this context, this article aims to evaluate the use of concrete fine fraction waste (CW-fine), with particles smaller than $2 \mathrm{~mm}$, to improve a soft subgrade soil of residual origin. For this purpose, their physical characteristics, the compaction and California Bearing Ratio (CBR) have been analyzed, both of the natural soil and of the mixtures with $\mathrm{CW}$-fine in proportions of 20,40 and $60 \%$ by weight. The results show that when adding $\mathrm{CW}$-fine, Atterberg limits, specific gravity of solid particles, optimum moisture content and expansion, decrease; while the maximum dry density and CBR increase. The expansion and $\mathrm{CBR}$ results of the soil mixture with $\mathrm{CW}$-fine show acceptable values for the subgrade reinforcement, in accordance with Brazilian paving standards.

Keywords: Subgrade stabilization. Concrete fine fraction waste.CBR

\section{Resumen}

El reaprovechamiento de los residuos de construcción y demolición (RCD) es una práctica sustentable que en los últimos años se viene investigando con gran intensidad alrededor del mundo. Los residuos de concreto son una parte de los RCD, cuya aplicación en pavimentos aún está en desarrollo. En ese contexto, este artículo pretende evaluar el uso de residuos de concreto de fracción fina ( $R C$-fino), con partículas menores a $2 \mathrm{~mm}$, para mejorar un suelo de subrasante blando de origen residual. Para tal finalidad, han sido analizadas sus características físicas, parámetros de compactación Relación de Soporte de California (del inglés California Bearing Ratio, CBR), tanto del suelo natural como de las mezclas con RC-fino en proporciones de 20,40 y $60 \%$ en peso. Los resultados muestran que al adicionar RC-fino, los valores de los límites de Atterberg, la densidad específica de los sólidos, el contenido de humedad óptimo y la expansión disminuyen; mientras que la densidad seca máxima y CBR aumentan. Los resultados de expansión y CBR de la mezcla de suelo con RC-fino, muestran valores aceptables para el refuerzo de subrasante, en conformidad con las normas brasileñas de carreteras.

Palabras Clave: Estabilización de subrasante. Residuo concreto fino.CBR 


\section{Introducción}

La industria de la construcción de 40 países, en el año 2012 ha sido responsable por una generación anual de más de $3 \mathrm{mil}$ millones de toneladas de residuos de construcción y demolición (RCD), siendo uno de los principales contribuyentes de emisión constante de dióxido de carbono a la atmósfera $[1,2]$.

De acuerdo con el Consejo Nacional de Medio Ambiente de Brasil, en su resolución $n^{\circ}$ 307, el 05 de julio del 2002, los RCD o popularmente llamados como escombros de obras, están compuestos por materiales generados en esta actividad, tales como: ladrillos, bloques cerámicos, concretos, acero, suelo, madera, pavimentos asfálticos, etc. [3-5]. En este país, la estimativa generada de RCD es $150 \mathrm{~kg} / \mathrm{m}^{2}$ de área construida y $600 \mathrm{~kg} / \mathrm{m}^{2}$ en demoliciones [4, 6]. De los cuales, los servicios de limpieza municipales recolectaron aproximadamente 122 mil toneladas de RCD/día en el 2018 [5]. Sin embargo, las cantidades generadas de residuos no corresponden al total de residuos producidos, debido a los aterros clandestinos que reciben una cantidad ignorada de escombros [7], así como otras formas clandestinas de deposición inadecuada. Las deposiciones irregulares son punto de proliferación de transmisores de enfermedades como Leptospirosis, además de generar contaminación, pudiendo ocasionar sedimentación y obstrucción en los recursos hídricos $[4,7,8]$.

En el modelo de construcción tradicional son normalmente utilizadas materias primas no renovables, y debido al crecimiento poblacional el consumo de residuos se transforma en un problema urbano [4]. Por lo que es importante la búsqueda de técnicas constructivas para materiales reciclados, más eficientes y menos agresivos con el entorno debido a las limitaciones del ecosistema [4, 9].

Para conseguir esta transformación del ciclo productivo, es necesario un concepto multidisciplinar, junto con cambios culturales, educación ambiental y sistemáticos [4]. Las investigaciones del sector académico han sido importantes para analizar las posibles aplicaciones de los residuos reciclados de concreto en pavimentos, a través del análisis de estabilización química y física de partículas de la fracción fina. Entre los investigadores de esta mencionada área encontramos: Kerni, Sonthwal y Jan [10] que utilizaron residuos de concreto $(\mathrm{RC})$ menores a $1.18 \mathrm{~mm}$ mejorando la densidad de empaque de masa del suelo y la capacidad de soporte; Paul y Cyrus [11] utilizaron RC menores a $20 \mathrm{~mm}$ mejorando el CBR de 3.5\% de suelo natural a $11.2 \%$ con $40 \%$ de RC, mientras Lovedeep, Shahbaz y Gill [12] utilizaron RC inferiores a 1.76 mm incrementando el CBR de $6.56 \%$ de suelo natural a $29.19 \%$ con $40 \%$ de residuos de concreto.

Al revisar informes de investigaciones geotécnicas de empresas privadas muestran algunas regiones del local de estudio, Foz do Iguazú-PR, que presentan suelos con baja capacidad de soporte, por lo que es necesaria una estabilización para el uso en pavimentación. Por otra parte, según datos de la municipalidad, solo en el año 2018 se registró una producción aproximada de 210 mil toneladas de residuos inertes, de los cuales aproximadamente el 80\% son RCD. El aprovechamiento de estos residuos en la estabilización de los suelos residuales blandos, en la región de Foz de Iguazú, ya sea en conjunto (el RCD), o por componente individual, tal como, el residuo de concreto (RC), 
es una alternativa de solución sustentable que necesita de mayores investigaciones para una futura aplicación.

En este contexto el presente trabajo tiene como finalidad evaluar la posibilidad de uso de los residuos de concreto de fracción fina (RC-fino) en la estabilización de suelos de subrasantes, contribuyendo al entendimiento para aplicación de RC-fino en suelos residuales de origen basáltico, como los existentes en Foz de Iguazú.

\section{Materiales y Métodos}

La metodología tiene carácter experimental y ha sido dividida en las siguientes etapas: adquisición del material, beneficiamiento del RC para obtener RC-fino, dosificación de suelo con los diferentes porcentajes de RC-fino, caracterización física y ejecución de los ensayos de compactación y CBR, tanto del suelo natural como de las mezclas.

Las muestras deformadas del suelo residual han sido extraídas en el Campo Experimental del Grupo de Pesquisa en Geotecnia de la UNILA (25²8'38.1"S,54³5'12.3”W), mediante una calicata de 80 centímetros de profundidad; han sido transportadas en sacos de tela y almacenadas en la cámara húmeda a una temperatura de 20 a $24^{\circ} \mathrm{C}$. Las muestras de RC han sido obtenidas en el vertedero de inertes de Foz de Iguazú $\left(25^{\circ} 27^{\prime} 48.1^{\prime \prime} \mathrm{S}, 54^{\circ} 36^{\prime} 22.3^{\prime \prime} \mathrm{W}\right)$, dando preferencia a escombros de concreto sin restos contaminantes, tales como: yeso y suelo; posteriormente almacenadas en sacos de tela y transportadas al Instituto Federal de Paraná (IFPR) para el proceso de beneficiamiento.

El beneficiamiento del residuo de concreto fue realizado con la Máquina de Abrasión de Los Ángeles, donde el material ha sido sujeto a choques con seis esferas de acero inoxidable por un tiempo de $6 \mathrm{hr}$, que tienden a reducir el tamaño de las partículas. El agregado resultante ha sido tamizado por una malla con abertura de $2 \mathrm{~mm}$, produciendo de esa forma el RC-fino. En la Figura 1 se presentan los materiales estudiados, finalmente preparados antes de la ejecución de los ensayos.

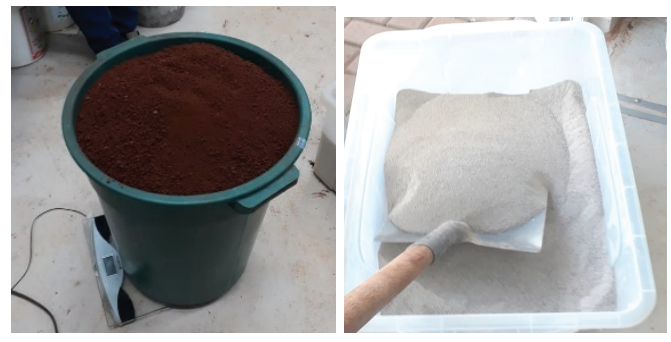

\section{Figure 1}

Materiales analizados: (a) suelo residual basaltico; (b) RC-fino.

Los ensayos de caracterización del suelo y suelo/RC-fino han sido realizados en conformidad con las Normas Técnicas Brasileras (NBR). Los ensayos realizados fueron: densidad específica de los sólidos (ס) [13], límite líquido (LL) [14] y límite plástico (LP) [15].

El ensayo de granulometría para las partículas finas ha sido realizado a través del granulómetro a láser de marca CILAS, modelo 1190, bajo la normativa ISO 13220 [16]. La lectura de los granos por el granulómetro comprende de 0.04 a $2500 \mu \mathrm{m}$ con un 
índice de oscuración medio de $25 \%$. Ha sido utilizada agua desionizada, con un tipo de dispersante que depende del pH de la muestra. Para muestras alcalinas se ha utilizado hexametafosfato de sodio a una concentración de $45.7 \mathrm{~g} / \mathrm{L}$, mientras para muestras ácidas se ha utilizado hidróxido de sodio con una concentración de 1 mol/L [17].

Han sido preparadas muestras de suelo con adición de 20, 40 y 60\% RC-fino. Las mezclas fueron cuantificadas en proporciones de peso de sólidos, para lo cual ha sido necesario la medición de las humedades higroscópicas iniciales del suelo y RC-fino, y a partir de estos valores se calculó el peso húmedo y la respectiva proporción del peso de sólidos de cada material.

El ensayo de compactación ha sido realizado de acuerdo con la NBR 7182 [18], usando un martillo automático de la marca $\mathrm{AMC}$, usando energía normal, cilindro pequeño y reutilización del material. Posteriormente se realiza el ensayo de CBR con base en la NBR 9895 [19] para todas las misturas, incluyendo el suelo natural. El control de la expansión del cuerpo de prueba sumergido en agua por 7 días, con lecturas en extensómetros cada $24 \mathrm{hr}$ [19]. El aumento del tiempo sumergido en agua se debe a la posibilidad de actividad cimentícia de los residuos de concreto fino.

Los sistemas de clasificación utilizados son el Sistema Unificado de Clasificación de Suelos (SUCS) y el sistema de la Asociación Americana de Carreteras Estatales y Transporte (AASHTO), detalles sobre estos sistemas pueden ser consultados en [20].

\section{Resultados}

Los valores de densidad específica de los sólidos y límites de Atterberg son presentados en la Figura 2. La densidad específica de los sólidos del suelo es $2.80 \mathrm{~g} / \mathrm{cm}^{3}$, mientras la del RC-fino es $2.62 \mathrm{~g} / \mathrm{cm}^{3}$. El incremento del porcentaje de RC-fino en las mezclas disminuye el valor de la densidad específica de los sólidos y los límites de Atterberg. La adición de $60 \%$ de residuo torna la mezcla no plástica.

En la Figura 3 se presentan las curvas granulométricas de los materiales estudiados, las mismas que muestran una tendencia al aumento de las partículas y disminución del porcentaje de finos con la adición de RC-fino, es decir se desplazan hacia la derecha y abajo.

Los resultados de clasificación por SUCS son los siguientes: el suelo es una arcilla de baja plasticidad con arena $(\mathrm{CL})$, y el residuo de concreto es una arena mal graduada con limo (SP-SM). Las mezclas con 20, 40 y $60 \%$ de residuo de concreto tienen la siguiente clasificación respectivamente: arcilla de baja plasticidad arenosa (CL), arcilla limo-arenosa (CL-ML) y arena limosa (SM). La clasificación del suelo el sistema AASHTO es A-7-6(13), y del RC-fino es A-3(0). Mientras las mezclas de suelo con 20, 40 y 60\% de residuo de concreto pertenecen a las clases A-6(7), A-4(3), y A-2-4(0).

La Figura 4, muestra los resultados de compactación de los materiales estudiados. Al adicionar residuos de concreto fino, las curvas tienden a aumentar la densidad seca máxima y disminuir el contenido de humedad óptima de las mezclas. 


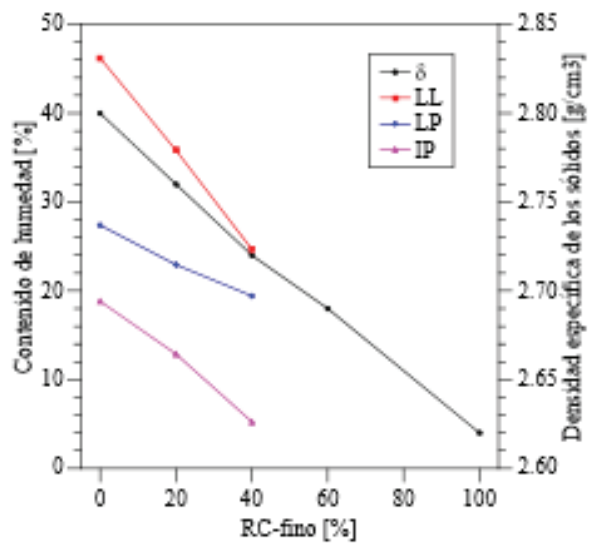

Figure 2

Densidad especifica de los sólidos y limites de Atterberg de las muestras.

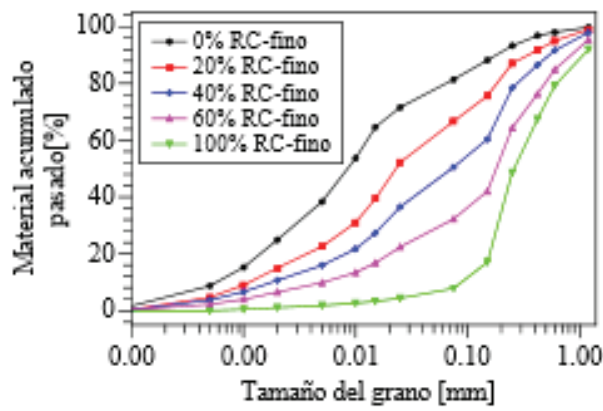

Figure 3

Curvas de granulometría de los materiales.

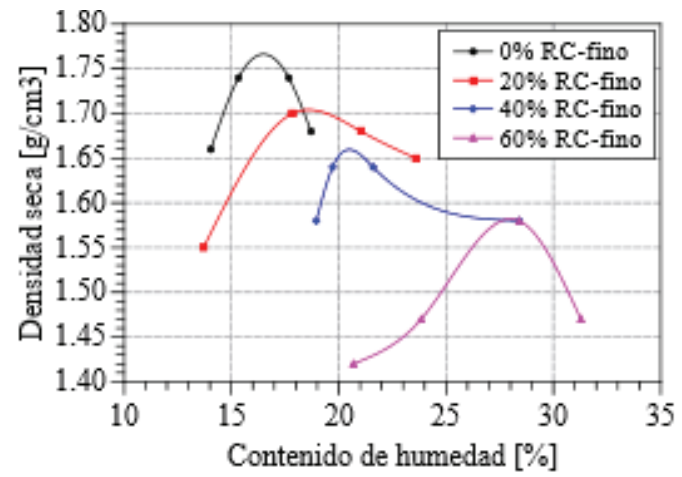

Figure 4

Compactación de las muestras.

En la Tabla 1 se muestra que la adición de residuos de concreto al suelo reduce el porcentaje de expansión, y con $60 \%$ de RC-fino adicionado la expansión es prácticamente nula.

En la Figura 5 se comparan los valores obtenidos del CBR de suelo con los diferentes porcentajes de residuos de concreto. Se observa que conforme se va aumentando el 
porcentaje de RC-fino aumenta el valor del CBR. En comparación con el CBR del suelo, existe un aumento de 6 veces con el 20\%, 12 veces con el $40 \%$, y 17 veces con el $60 \%$ de RC-fino. Mayores detalles obre los calculos del CBR se encuentran en [21].

\section{Table 1}

Verificación de la expansión de los materiales.

RCD-C fino [\%]
0
20
40
60

Expansión [\%]
1.23
0.34
0.13
0.01

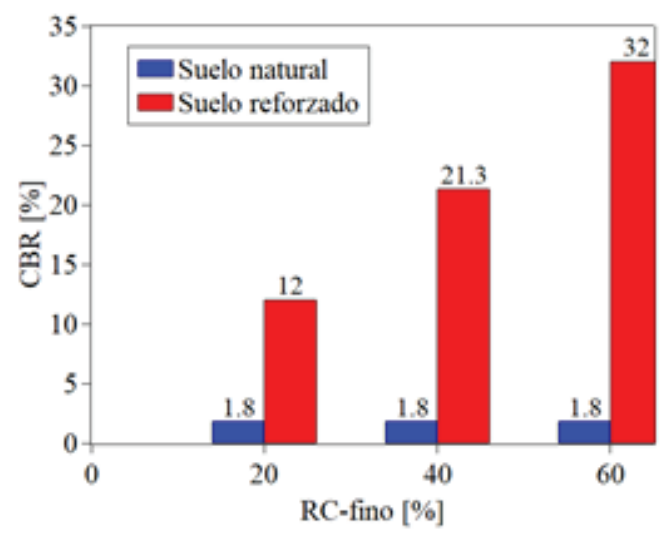

Figure 5

Comparación entre los valores de CBR.

\section{Discusión}

En la literatura varía el resultado de la densidad específica de los sólidos del RC debido a la heterogeneidad del material y tamaño de las partículas que están siendo analizadas, como se muestran en las investigaciones (2) y [22]. El valor encontrado para RC-fino se encuentra dentro del intervalo de la literatura revisada. No obstante, al mezclar el RCfino con el suelo, el comportamiento va a depender de la proximidad de las densidades específicas de los sólidos de los materiales originales, pudiendo generar una variación mínima o lineal de este parámetro [23].

La tendencia de reducción del límite líquido y el índice de plasticidad con la adición de residuos de concreto fino ha sido observado en la literatura, por ejemplo, en [23]. Este efecto, también es encontrado al adicionar cemento al suelo [24]. Al parecer el cemento tiene la acción de reducir la plasticidad de los suelos arcillosos, y el RC-fino puede contener fracciones de cemento, que justificaría su acción estabilizadora.

A pesar del proceso de beneficiamiento del residuo de concreto, las partículas de RC-fino son mayores al del suelo analizado, lo que explica la tendencia de las curvas granulométricas al incremento de partículas gruesas y disminución de porcentaje de 
finos (limo y arcilla) con la adición de RC-fino, un ejemplo similar se encuentra en [23]. Sin embargo, esta característica no se observa cuando la curva de distribución granulométrica del suelo y el RC-fino, al presentar poca diferencia [25].

Según la clasificación de AASTHO el suelo presenta características de regular a malo para ser aplicado como subrasante. Sin embargo, al adicionar RC-fino este calificativo pasa a ser de excelente a bueno. Los parámetros reducidos de plasticidad y aumento del tamaño de las partículas, contribuye a mejores condiciones como material de subrasante.

El aumento en la densidad seca máxima y una disminución del óptimo contenido de humedad con la adición de RC-fino, puede estar relacionado con una mejoría en las condiciones de compactación cuando existen una granulometría más graduada. Comportamientos similares han sido observados en la literatura, por ejemplo, en [23]. La adición de cemento en la mezcla con suelo también presenta un comportamiento semejante, según el trabajo de [24]. Sin embargo, existe resultados que muestran comportamientos diferentes cuando la adición de residuos de concreto es de 50 y $60 \%$ [11].

La expansión del suelo cumple con el valor mínimo exigido por las normas brasileñas de transporte, que es de $2 \%$ como mínimo [26]. La reducción de la expansión por efecto de la adición de RC-fino al suelo fue observado también al usar cemento como agente estabilizador de subrasante, por ejemplo [24]. La reducción de este parámetro puede estar relacionada a efectos de estabilización química del RC-fino durante los siete días de cura, potencialmente generada por partículas remanentes del cemento presentes en el residuo de concreto fino que al ser hidratadas reaccionan con las partículas del agua, generando un endurecimiento en toda la mezcla [10-12].

El suelo estudiado no cumple con las exigencias mínimas de CBR para materiales de subrasante, establecidas por las normas brasileñas de transporte [26]. No obstante, al adicionar RC-fino, el material satisface los requisitos, y viabiliza su uso como subrasante, es decir funciona como un aditivo estabilizador, así como el cemento o la cal, que fueron estudiados en [26]. Tendencias similares han sido observadas en la literatura, como es presentado por [11, 12]. Resultados opuestos, es decir, disminución del CBR al incrementar residuo, fueron encontrados con $40 \%$ de RCD [27]. El incremento del CBR puede estar relacionada la estabilización química cuando existe material de cemento activo en el interior de los residuos de concreto, y física cuando la distribución granulométrica favorece las condiciones de compactación.

\section{Conclusiones}

El suelo residual estudiado ha sido clasificado con características pobres para ser utilizado como subrasante. La adición de residuo de concreto de fracción fina (RC-fino) al mencionado suelo, mejora sus propriedades de expansión, CBR, y otras. Viabilizando su aplicación como material de subrasante, de acuerdo con las normas brasileñas (DNIT). Los estudios abarcan adiciones de residuos de concreto de $20 \%$ a más; adiciones menores todavía no fueron investigadas. Futuras investigaciones pueden incluir análisis 
de viabilidad económica, análisis mineralógicos y la ejecución de ensayos con tiempo de cura mayores.

\section{Agradecimientos}

Este trabajo forma parte del proyecto de investigación PID2530-2020 de la UNILA. Los autores agradecen la colaboración de las siguientes instituciones: Instituto Federal de Paraná (Campus Foz de Iguazú) y Colegio Estadual Prof. Flavio Warken.

\section{Conflicto de Intereses}

Los autores declaran que no existen conflicto de intereses en la publicación.

\section{References}

[1] Akhtar A, Sarmah A. Construction and demolition waste generation and properties of recycled aggregate concrete: A global perspective. Journal of Cleaner Production. 2018;186:262-281.

[2] Oliveira T, Dezen B, Possan E. Use of concrete fine fraction waste as a replacement of Portland cement. Journal of Cleaner Production. 2020;123-126.

[3] Resolução n 4672015.

[4] John V, Agopyan V. Reciclagem de resíduos da construção. Paper presented at: Seminário- Reciclagem de Resíduos Sólidos Domiciliares; 2000; São Paulo, Brazil.

[5] Associação Brsileira de Empresas de Limpeza Pública e Resíduos Especiais. Panorama dos resíduos sólidos no Brasil 2018/2019. 2019.

[6] Pinto T. Metodologia para a gestão diferenciada de resíduos sólidos da construção urbana [Doctoral Thesis]. São Paulo: USP; 1999.

[7] Schneider D. Deposições irregulares de resíduos da construção civil na cidade de São Paulo [tesis de maestría]. São Paulo: USP; 2003.

[8] Motta R. Estudo laboratorial de agregado reciclado de resíduo sólido da construção civil para aplicação em pavimentação de baixo volume de tráfego [Doctoral Thesis]. São Paulo: USP; 2005.

[9] Soncim S, Júnior G, Júnior M, de Almeida M, de Almeida S, Vidal F. O emprego do resíduo da reciclagem de garrafas PET (Polietileno Tereftalato) como agregado em reforço de subleitos de rodovias. Paper presented at: IV Simpósio Internacional de Qualidade Ambiental; 2004; Porto Alegre, Brazil.

[10] Kerni V, Sonthwal V, Jan U. Review on stabilization of clayey soil using fines obtained from demolished concrete structures. International Journal of Innovative Research in Science, Engineering and Technology. 2015;4:296-299.

[11] Paul H, Cyrus S. Stabilization of Weak Subgrade Soil Using Demolished Concrete Aggregate. Indian Geotechnical Conference IGC. 2016.

[12] Singh L, Singh S, Gill K. Improvement in CBR value of soil using waste concrete fines. International Journal of Science Technology \& Engineering. 2017;3.

[13] Associação Brasileira de Normas Técnicas. ABNT NBR 6508: Grãos de solos que passam na peneira de 4.8mm: Determinação da massa específica. 1984.

[14] Associação Brasileira de Normas Técnicas. ABNT NBR 6459: Solo: Determinação do limite de liquidez. 2016.

[15] Associação Brasileira de Normas Técnicas. ABNT NBR 7180: Solo: Determinação do limite de plasticidade. 2016.

[16] International Organization for Standardization. ISO 13220: Particle size analysis: Laser diffraction methods. 2009.

[17] Mauri J, Ruiz H, Fernandes R, Ker J, Rezende L. Dispersantes químicos na análise granulométrica de latossolos. Revista Brasileira de Ciência do Solo. 2011;35:1277-1284.

[18] Associação Brasileira de Normas Técnicas. ABNT NBR 7182: Solo - Ensaio de compactação. 2016.

[19] Associação Brasileira de Normas Técnicas. ABNT NBR 9895: Índice de Suporte de California: Requisitos. 2016. 
[20] Das B. Fundamentos de ingeniería de cimentaciones. $7^{\text {th }}$ ed. México: Cengage Learning; 2012.

[21] Ochoa AS. Estudio experimental sobre la estabilización de una subrasante limo-arcillosa con RCDconcreto fino (partículas $<2 \mathrm{~mm}$ ) para aplicación en pavimentos [Trabajo de Conclusión de Curso]. Foz de Iguazú: UNILA; 2019.

[22] Favaretto P, Hidalgo G, Sampaio C, Silva R, Lermen R. Characterization and use of construction and demolition waste from South of Brazil in the production of foamed concrete blocks. Applied Sciences. 2017;7:1090.

[23] Silva A, Fucale S, Ferreira S. Efeito da adição de resíduos da construção e demolição (RCD) nas propriedades hidromecânicas de um solo areno-argiloso. Matéria. 2019;24.

[24] Joaquim A. Estudo de dois solos tropicais melhorados com cimento ou cal para emprego em camadas superiores de estradas não pavimentadas [Master's thesis]. Campinas: UNICAMP; 2017.

[25] Santos, E. Aplicação de resíduos de construção e demolição reciclado (RCD-R) em estruturas de solo reforçado [Tesis doctoral]. São Paulo: USP; 2007.

[26] Departamento Nacional de Infra-estrutura de Transportes (DNIT). Manual de pavimentação. 2006.

[27] Luz C, da Silva SN, da Silva SN. Análise da mistura do agregado reciclado de RCD associado ao solo laterítico para utilização na camada de base de pavimentos. Paper presented at: $9^{\text {th }}$ Forum Internacional de Resíduos Sólidos; 2019 June 13-15; Porto Alegre, Brazil. 